\title{
Erythrodermic Psoriasis with Superimposed Fungal Infection-a Presentation in the Emergency Room
}

\author{
Muhammad Akbar Baig (D), Sadaf Sheikh (D) \\ Department of Emergency Medicine, Aga Khan University Hospital, Karachi, Pakistan
}

Cite this article as: Baig MA, Sheikh S. Erythrodermic Psoriasis with Superimposed Fungal Infection-a Presentation in the Emergency Room. Eurasian J Emerg Med. 2018; 17: 33-5.

\begin{abstract}
Erythrodermic psoriasis or erythroderma is a life-threatening, generalized exfoliative dermatitis involving majority of the body surface. A detailed evaluation is required for the identification of its underlying cause to successfully treat and prevent its relapse. We present a case of erythroderma in our local setting and review this disease in terms of the importance of immediate diagnosis and therapy for improved patient survival.
\end{abstract}

Keywords: Erythrodermic psoriasis, erythroderma, emergency department, Pakistan

\section{Introduction}

Erythrodermic psoriasis/erythroderma is a severe variant of psoriasis, which poses a life-threatening risk to the patient and a treatment challenge to the physician. It tends to involve approximately $90 \%$ of the body surface, giving rise to erythema and scaling. Uncontrolled flares of the disease can lead to superimposed infections involving the skin areas already afflicted with the condition. There is a paucity of relevant information regarding this condition for an emergency physician; therefore, we present a case of erythroderma with superimposed fungal skin infection encountered by us in the emergency department.

\section{Case Presentation}

A 62-year-old man presented to the emergency department with the complaint of acute eruption of fiery red skin lesions with intense pain and itching. He was a known case of asthma, hypothyroidism, and psoriasis (disease in remission). Approximately 2 weeks prior to this presentation, he had been using a homeopathic medication for his overall general health, after which he noticed an increased erythematous plaque formation occupying his arms, torso, and legs. On initial assessment, the patient exhibited stable vital signs. Skin examination revealed erythematous geographical plaques with well-defined margins and superficial skin exfoliation that was distributed along the flexor and extensor aspects of bilateral forearms, the anterior aspect of the abdomen, bilateral medial thighs, and the posterior aspect of his lower legs. The lesions were warm and exquisitely tender to touch (Figures 1, 2); furthermore, clear fluid extravasation was seen. A provisional diagnosis of erythrodermic psoriasis was made. The patient was managed in the emergency department with intravenous fluids, analgesics, and a topical antihistamine lotion. His complete blood count and serum electrolyte levels were unremarkable as shown by laboratory test results. The patient was admitted to the medical ward and reviewed by a skin specialist the next day. Potassium hydroxide staining of the superficial skin scrapings demonstrated pseudo hy-

ORCID IDs of the authors: M.A.B. 0000-0002-2830-9099; S.S. 0000-0001-7457-0012. 


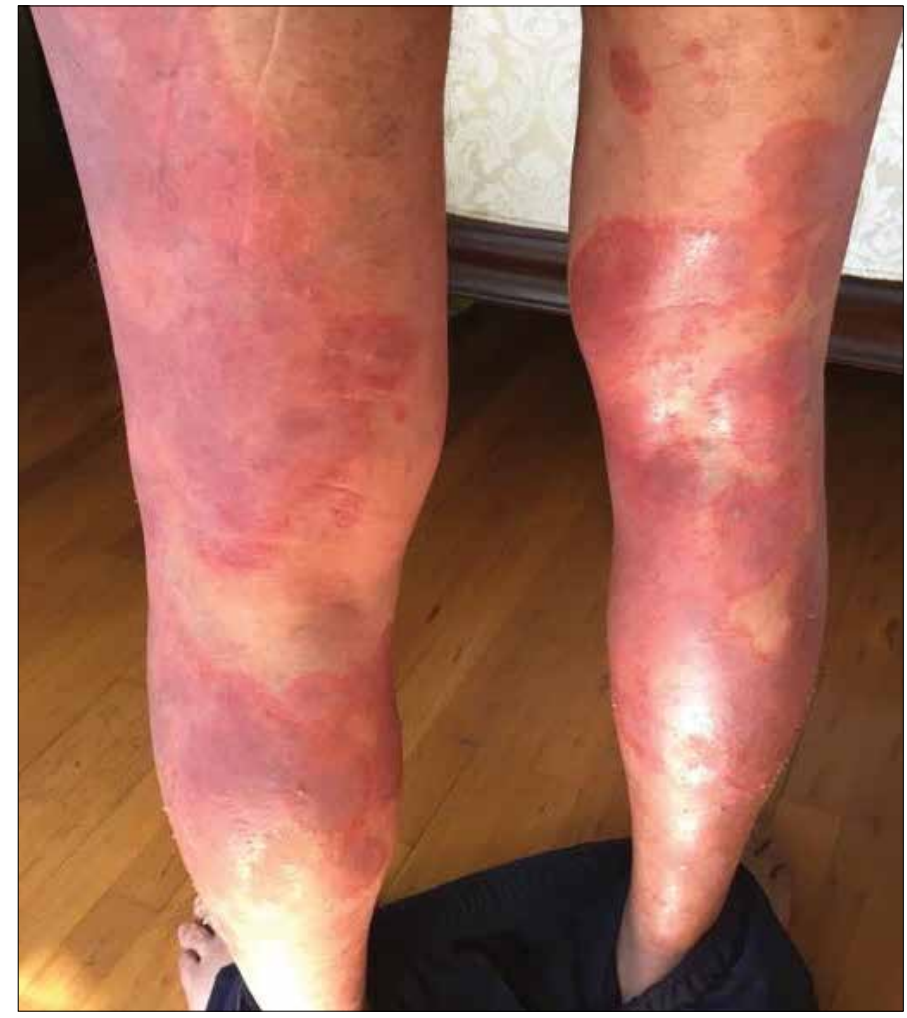

Figure 1. A 62-year-old male with erythrodermic psoriasis

phae formation, indicating a fungal infection. Therefore, oral antifungal fluconazole was started; topical and oral steroids were used as well, and the patient demonstrated a significant improvement during the course of his stay. The patient is currently admitted and is on oral antifungals as well as oral and topical steroids; he is due for discharge with advice for follow upin dermatology clinic.

\section{Discussion}

Erythrodermic psoriasis is an autoimmune condition affecting $3 \%$ of the world population, and it can be life threatening (1). In general, the condition is known to predominantly affect male population with a male to female ratio of $4: 1$; the mean age of the affected patients ranges from 40 to 60 years (2). However, no study has correctly estimated the current status of the disease in Third World nations such as Pakistan.

Erythrodermic psoriasis can be a challenging condition to diagnose, especially for the emergency physicians who have a limited experience in the management of this disease. Majority of such patients are misdiagnosed with drug reaction, cutaneous T-cell lymphoma, or atopic dermatitis (as in our presented case) (3). Dermatosis (established in a patient), like psoriasis, is the most common cause of erythrodermic psoriasis (4). It may also occur in relation to withdrawal of steroids, use of systemic or herbal medications, burns, and systemic illnesses (5). The most common precipitating cause of erythrodermic psoriasis is withdrawal of systemic corticosteroids. Thus, the use of systemic corticosteroids in patients with psoriasis is avoided as much as possible (6).

The underlying mechanism of erythrodermic psoriasis involves $T$ cells playing a central role in the pathogenesis, which includes Lang-

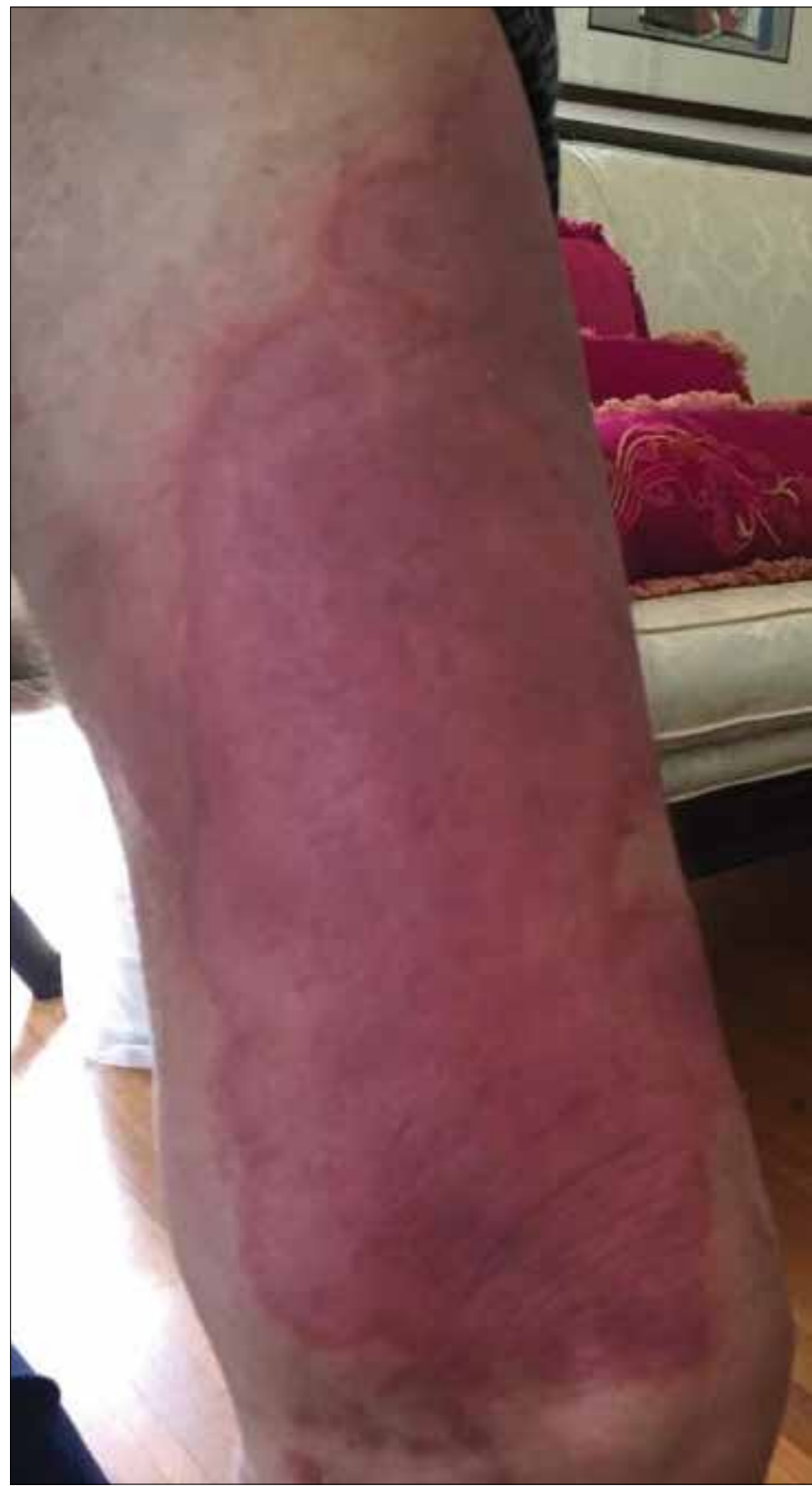

Figure 2. Erythrodermic psoriasis demonstrated along the anteromedial aspect of the left thigh

erhans cell activation and maturation by skin antigens, $\mathrm{T}$ cell activation by the mature Langerhans cells, expansion and differentiation of the $T$ cells within the lymph nodes, transport of the activated $T$ cells from lymph node to the skin, and the subsequent release of cytokines. These cytokines are responsible for epidermal and vascular changes with pro-inflammatory effects (7).

Erythrodermic psoriasis causes development of erythematous patches, which coalesce to form an extensive erythema and involve most of the skin. The skin induration progresses to attain a dull gray discoloration and gives a sensation of skin tightness to the patient. A concomitant presence of lymphadenopathy and/or hepatosplenomegaly should raise the suspicion of drug reaction or malignancy and should be accordingly investigated (5). 
The initial management of erythrodermic psoriasis includes replacement of nutritional, fluid, and electrolyte depletion. Local skin care should be performed using application of emollients and mildly potent corticosteroids along with sterile dressings. Any identified precipitant should be avoided, and the underlying cause should be treated to achieve a complete cure. Secondary fungal or bacterial infections are treated using recommended antifungals or antibiotics, respectively, tailored specifically as per local hospital guidelines. Any hemodynamic instability should be immediately addressed. Disease outcome is unpredictable and may include multiple exacerbations necessitating the use of systemic corticosteroids. Other medications such as methotrexate, acitretin (Soriatane), or cyclosporine may also be considered $(8,9)$. Skin inflammation is a predominant feature of erythroderma, and rapid release of tumor necrosis factor-alpha (TNF-a) causes the flare. Therefore, TNF-a blocking biologics such as etanercept, adalimumab, and infliximab that target this specific pathway can be considered for treatment; however, they should be used after a careful consideration of the associated side effects (10).

There is conflicting evidence regarding prognosis of erythroderma in developing nations. Common causes of death in affected patients are pneumonia, sepsis, and congestive heart failure. The elderly population is at a higher risk due to these complications; however, the incidence of death has substantially reduced due to early diagnosis and therapy (11).

\section{Conclusion}

Erythrodermic psoriasis is a rare and fatal exfoliative dermatitis, which should be immediately recognized and promptly treated. The mainstay of treatment is founded on aggressive supportive care and use of anti-inflammatory immunosuppressive therapy including the newer biologic agents.

We presented the case of a patient in whom erythroderma was likely triggered by the use of homeopathic medicines and was successfully treated with steroids and antifungals. Our patient's global assessment and compliance with treatment was good; therefore, he had a favorable recovery from the condition.
Informed Consent: Written informed consent was obtained from patient who participated in this case.

Peer-review: Externally peer-reviewed.

Author Contributions: Concept - M.A.B., S.S.; Supervision - M.A.B., S.S.; Resources - M.A.B., S.S.; Materials - M.A.B., S.S.; Literature Search - M.A.B., S.S.; Writing Manuscript - M.A.B., S.S.; Critical Review - M.A.B., S.S.; Other - M.A.B., S.S.

Conflict of Interest: No conflict of interest was declared by the authors.

Financial Disclosure: The authors declared that this study has received no financial support.

\section{References}

1. Schön MP. Advances in psoriasis treatment. Lancet. $2005 ; 366: 1333-5$. [CrossRef]

2. Sehgal VN, Srivastava G. Exfoliative dermatitis: A prospective study of 80 patients. Dermatologica. 1986; 173: 278-84. [CrossRef]

3. Balasubramaniam P, Berth-Jones J. Erythroderma: $90 \%$ skin failure. Hosp Med. 2004;65:100-2. [CrossRef]

4. Pal S, Hamon TS. Erythroderma: A clinico-etiologic study of 90 cases. Int J Dennatol. 1998; 37: 104-7.

5. Rubins AY, Hartmane IV, Liebriedis YM, Schwartz RA. Therapeutic options for erythrodenna. Cutis. 1992; 49: 424-6.

6. Stinco G, Errichetti E. Erythrodermic psoriasis: current and future role of biologicals. Bio Drugs. 2015; 29: 91-101. [CrossRef]

7. Wilson DC, Jester JD, King LE., Jr. Erythrodenna and exfoliative dermatitis. Clin Dennatol. 1993; 11: 67-72.

8. Karakayli G, Beckham G, Orengo I, Rosen T. Exfoliative dennatitis. Am Fam Physician. 1999; 59: 625-30.

9. Sehgal VN, Srivastava G, Sardana K. Erythroderma/exfoliative dermatitis: A synopsis. Int J Dermatol. 2004; 43: 39-47. [CrossRef]

10. Rosenbach M, Hsu S, Korman NJ, Lebwohl MG, Young M, Bebo BF Jr, et al. Treatment of erythrodermic psoriasis: from the medical board of the National Psoriasis Foundation. J Am Acad Dermatol. 2010; 62: 655-62. [CrossRef]

11. Nicolis GD, Helwig EB. Exfoliative dermatitis: A clinicopathologic study of 135 cases. Arch Dermatol. 1973; 108: 788-97. [CrossRef] 